\title{
BUCHHALTUNGSAUTOMATISIERUNG UND DIE ZUKUNFT DES BUCHHALTUNGSBERUFS
}

\section{ORIGINALER ARTIKEL}

SUMAR, Ramiro Rodrigues ${ }^{1}$

SUMAR, Ramiro Rodrigues. Buchhaltungsautomatisierung und die Zukunft des Buchhaltungsberufs. Revista Científica Multidisciplinar Núcleo do Conhecimento. Jahrgang 06, Ed. 06, Vol. 17, S. 167-181. Juni 2021. ISSN: 2448-0959, Zugangslink: https://www.nucleodoconhecimento.com.br/buchhaltung/buchhaltungsberufs, DOI: 10.32749/nucleodoconhecimento.com.br/buchhaltung/buchhaltungsberufs

\section{ZUSAMMENFASSUNG}

Das Vorhandensein von Qualität in den erbrachten Dienstleistungen ist in der Tat für das Überleben des Buchhaltungsberufs unerlässlich, da es sich in das Ziel eines Buchhalters übersetzt. Informationssysteme, neue Technologien der künstlichen Intelligenz und Innovationen können, wenn sie mit dem Fachwissen von Buchhaltungsfachleuten verbunden sind, zu einer höheren Leistung der Unternehmen und folglich der Wirtschaft führen. In Anbetracht der Tatsache, dass der Beruf des Buchhalters versucht, den Steuerbehörden Steuerinformationen zur Verfügung zu stellen, zielt dieser Artikel darauf ab, zu zeigen, dass sich dieser Beruf in einem Migrationsprozess in Bezug auf die Automatisierung von Aufgaben befindet, um ein Managementinstrument zu werden, das in der Lage ist, bei der durchsetzungsfähigen Entscheidungsfindung zu helfen, um zur Erfüllung der sozialen Verantwortung der Unternehmen beizutragen. Die Problematisierung dieser Studie basiert auf der folgenden Problemfrage: Welche Rolle wird der Buchhalter bei der Automatisierung der Buchhaltung spielen? Wird der Beruf des Buchhalters enden, wie vom Sapiens Institute in der Forschung von 2018 vorhergesagt? Um die Ziele zu erreichen, wurde

\footnotetext{
${ }^{1}$ Master-Abschluss in Kontrolle, Spezialist für Technologiemanagement, Strategische Verwaltung, Informationssicherheit und Abschluss in Rechnungswissenschaften. 
die Literaturrecherche genutzt. Als Ergebnis zeigte der Artikel die Rolle des Buchhalters im Prozess der Entwicklung und des Wachstums von Unternehmen vor und nach der Automatisierung bürokratischer Prozesse sowie die Perspektive des Zukunftsszenarios des Berufs. So wurde festgestellt, dass der Buchhalterberuf einen plötzlichen Positionswechsel erfährt, so dass er als Beruf überlebt und seine Rolle in der Gesellschaft und Wirtschaft des Landes beibehält.

Schlüsselwörter: Buchhaltungsautomatisierung, Buchhaltungsfachmann, Soziale Verantwortung, Entscheidungsfindung.

\section{EINLEITUNG}

Es gibt ein Szenario großer Veränderungen auf dem Buchhaltungsmarkt mit neuen Technologien, dem Einsatz künstlicher Intelligenz und Prozessautomatisierung. Und mit diesen Veränderungen gehen die Sorgen, Ängste und Unsicherheiten ein, die das Neue bei den Menschen hervorruft (SOUZA et al., 2018).

Diese Veränderungen im Land werden von allen gesehen und gefühlt, wo die Inflation nicht mehr als Problem angesehen wird, die Arbeitslosenquoten gesunken sind, der Zugang zu Krediten und Konsum zugenommen hat und infolgedessen die Armutsraten gesunken sind (CUNHA, 2019).

Daher gibt es ein unternehmerisches Szenario, in dem jeden Tag neue Unternehmen entstehen und mehr Fachkräfte gefragt werden, aber einige Berufe laufen Gefahr, aufgrund von Technologie und Automatisierung nicht zu existieren (LEITE, 2017; ARAÚJO, 2020).

Laut Rozenbaum (2018) zeigt eine Studie des französischen Instituts Sapiens aus dem Jahr 2018, dass fünf Berufe vom Aussterben bedroht sind, darunter der Buchhalter, der in einem Büro ohne Zugang zu Technologien wie dem Einsatz von Computern arbeitet .

Der moderne Buchhalter muss sich immer noch mit dem bürokratischen Teil, den Gesetzmäßigkeiten und der großen Menge an Papieren auseinandersetzen, die Platz 
einnehmen. Da die Automatisierung von Prozessen und andere Fortschritte, die die Arbeit der Buchhaltung erleichtern, auf Technologien zurückzuführen sind, müssen sich die Buchhaltungsfachleute an die Nutzung der Technologie zu ihren Gunsten anpassen oder sich nur ungern ändern und sehen, wie ihr Beruf angesichts der Automatisierung verschwindet ( DINIZ, 2014).

Laut der Studie des französischen Instituts werden eine Zahl von über zwei Millionen Menschen "in den kommenden Jahren mit großer Wahrscheinlichkeit ihre Arbeitsplätze verschwinden sehen". Die Daten für die Studie stammen vom DARES (Statistical Studies Service des französischen Arbeitsministeriums) und darin ist beispielsweise zu beobachten, dass Bankangestellte bis 2050 und Buchhalter im Jahr 2056 vollständig verschwinden können (ROZENBAUM, 2018).

Die Wettbewerbsfähigkeit auf dem Markt, mit Unternehmern, die jeden Tag nach Innovation und neuen Technologien suchen, um ihr Geschäft zu erweitern, macht den Buchhaltungsprofi in diesem Umfeld eingefügt. Unternehmen, die mit dem wettbewerbsintensiven Markt konfrontiert sind, können nicht umhin, neben der Hilfe des Buchhalters zu haben, da dieser Fachmann in seiner Zuschreibung Qualität und Effizienz in allen Bereichen des Geschäfts anstreben (CHIAVENATO, 2004).

Ein weiterer Punkt ist, dass die Generation der Millennials den Arbeitsmarkt übernimmt und Positionen in der Unternehmenshierarchie und Entscheidungsfindung gewinnt. Für den Buchhalter ist es daher wichtig, diese Öffentlichkeit zu kennen und zu verstehen, die Agilität und Qualität von Buchhaltungsdienstleistungen erwartet.

Der Zweck dieses Artikels ist es jedoch, die Zukunft des Buchhaltungsberufs und die Automatisierung, die in der Buchhaltungsarbeit stattgefunden hat, zu untersuchen. Diese Veränderungen sind notwendig, um den Buchhalter auf dem wettbewerbsorientierten Markt zu halten, sowie seine Bedeutung für die Schaffung neuer Arbeitsplätze, seine Fähigkeit zu unternehmen und zu innovieren, sowie die soziale Bedeutung vor seiner buchhalterischen und steuerlichen Funktion. 
Der Buchhalter durchläuft große Veränderungen, ist nicht mehr der Profi, der nur Probleme und Konten bringt, und fungiert als Unterstützung bei der Verwaltung von Unternehmen. Zu diesem Zweck muss sich der Fachmann an die neuen Anforderungen anpassen und innovativ sein, um seine Rolle und Bedeutung nicht nur für Unternehmen und Manager, sondern auch für die Gesellschaft nach seiner sozialen Rolle zu erhalten.

Die Methodik, die beim Schreiben dieses Artikels verwendet wurde, war bibliographische Forschung, durch Annais, Forschung, Bücher, Zeitschriften, InternetInterviews, Internet-Artikel und veröffentlichte akademische Artikel. Für Gil (2008) wird bibliographische Forschung auf der Grundlage von bereits ausgearbeitetem Material entwickelt, das hauptsächlich aus Monographien, Dissertationen, wissenschaftlichen Artikeln und Büchern besteht.

Wenn es um den Zweck geht, erklären Marconi und Lakatos (1992), dass bibliographische Forschung dem Betrachter die Möglichkeit gibt, direkt mit den Materialien in Kontakt zu treten, die zum Thema der Forschung geschrieben wurden, und den Forscher bei seiner Analyse oder zum Zeitpunkt der Manipulation der Informationen zu unterstützen. Bibliographische Forschung gilt als erster Schritt zur Initiierung wissenschaftlicher Forschung.

\section{THEORETISCHER RAHMEN}

\subsection{GESCHICHTE DES BUCHHALTERS}

Es gibt Berichte, dass die Figur des Buchhalters seit Beginn der Zivilisation präsent ist. Laut ludícibus und Marion (2006, S. 32): "[...] schon seit den primitivsten Völkern existierte Buchhaltung aufgrund der Notwendigkeit, das Familienerbe zu kontrollieren, zu messen und zu erhalten und sogar aufgrund des Austauschs von Gütern zur größeren Befriedigung von Leuten [...]".

Trotz der Berichte war die offizielle Existenz der Buchhaltung in der Neuzeit, im 15. bis 16. Jahrhundert, in der Region Italien gekennzeichnet. In der Renaissance gewann 
der Beruf des Buchhalters an Raum in Bereichen wie: Wirtschaft, Kunst, Wissenschaft und Technologien (FIGUEIREDO; STRASSBURG, 2008).

In Brasilien, noch bevor die ersten kommerziellen technischen Schulen auftauchten, wurde die Buchhaltung von der Figur des "Buchhalters" praktiziert, einem nach dem Handelsgesetzbuch von 1850 definierten Fachmann, der sich laut Francisco D'Auria (1949) um die Transaktionen der kommerziellen Einrichtungen zu dieser Zeit kümmerte und aufzeichnete (CARVALHO, 2017).

Die Entwicklung des Buchhaltungsberufs folgte der Verabschiedung des Gesetzesdekrets Nr. 9.295 vom 27. Mai 1946, das den Bundesrechnungsrat schuf und die Zuschreibungen von Buchhaltern, Buchhaltungstechnikern und Buchhaltern definierte (DOS SANTOS et al., 2019).

Mit dem Rat erweiterte sich der Beruf und viele Probleme traten mit der Gleichstellung von Fachleuten mit unterschiedlichem Bildungs- und Wissensstand auf. Wenn es auf der einen Seite den Buchhalter gibt, der mit einem Grundstudium ausgebildet wurde, koexistierte ein Fachmann ohne Jegliches Studium im selben Beruf (NETO, 2010).

Dies führte zu dem Desinteresse junger Menschen an der Buchhaltung, da der Status dieses Berufs nicht derselbe war wie der anderer Formationen wie Recht und Wirtschaft. Wie kann man das Interesse an einem Beruf wecken, der ebenen von Fachleuten gegeneinander stellt? Dies war nur mit einem Nachweis der Kompetenz und der Trennung vom Buchhaltungstechniker zum Buchhalter selbst möglich.

1972 wurde die Prüfung durch den Beschluss Nr. 220 sowie die Rundschreiben Nr. 178 und Nr. 179 der brasilianischen Zentralbank geregelt. Es wurden demonstrative Standards festgelegt, die verlangten, dass Fachleute an ein Audit durch zertifizierte Personen angepasst werden mussten (ABREU, 2013; CORDEIRO, 2011).

Der Berufsstand des Buchhalters erhielt jedoch erst im Dezember 1976 mit der Verabschiedung des Gesetzes 6.404, das als SA-Gesetz (Sociedades Anônimas Anonyme Gesellschaft) bekannt ist, einen Status. Aus diesem Gesetz entstand die Brasilianische Wertpapierkommission (CVM), die die Zentralbank in Bezug auf die 
Abgabe von Gutachten und einen Teil der Rechnungslegungsvorschriften sowie die Kontrolle des Kapitalmarkts in Brasilien ersetzt hat (CARVALHOSA, 2017).

Für Santos $(2008$, S. 9)

[...] in den letzten Jahren, insbesondere nach dem Ende der Ära der hohen Inflation, ab Mitte der 1990er Jahre, hat es eine Periode großer Veränderungen und Anpassungen durchgemacht. Unternehmen, die ihren Fokus agiler auf ihr Kerngeschäft, ihr Kerngeschäft, anpassten und nicht mehr mit inflationären Gewinnen und Umsätzen umgingen, erzielten eine höhere Erfolgsquote. Und in diesem Moment hat der Buchhaltungsprofi eine primäre Beteiligung an der Bereitstellung von Steuer-, Unternehmens- und Finanzberatung. [...]. Der Zähler ist zunehmend der Kompass des Unternehmens.

Es wird beobachtet, dass sich die Fähigkeiten und Fähigkeitenanforderungen des Buchhaltungsprofis auf Strategien und Entscheidungsfindung in Unternehmen konzentrieren. Aufgrund des technologischen Wandels befinden sich Regierungen, Unternehmen und Manager in einem hohen Innovationsgrad, der zunehmend von den Buchhaltungsfachkräften verlangt wird, die wiederum die Entscheidungsfindung unterstützen (DE OLIVEIRA REIS et al., 2015).

In einem hart umkämpften und agilen Umfeld ist die sichere Beschaffung von Informationen ein Unterschied. Genauigkeit und Zuverlässigkeit werden auch von modernen Unternehmen verlangt, die ihr Überleben auf dem Markt sichern und den Buchhalter zu einem Vermögensdatenwissenschaftler und Buchhaltungseinträgen machen wollen, so dass er wissen muss, wie man die Daten interpretiert und Strategien und Wege skizziert, die bessere Ergebnisse gewährleisten. All diese Forderungen kommen dem entgegen, was Santos (2008, S. 10) in seinem Buch schreibt:

[...] Der Zähler sollte nicht vor dem Hintergrund verlieren, dass die Buchhaltung nicht dafür ist, sondern für den Benutzer. Buchhaltung ist die Sprache des Geschäfts und diese Sprache erzählt die Geschichte jedes Unternehmens. Die Perfektionierung dieser Sprache, um ihre Vorhersagekraft und ihren Nutzen für den Benutzer zu erhöhen, ist vielleicht die Hauptaufgabe des Buchhalters. [...] 
Daher muss der Fachmann im Prozess des Lernens, der Aktualisierung und der Ausbildung sein, um ein Experte in seiner Rolle und bei der Beschaffung von Daten zu sein, die dem Unternehmen bei seinem sozialen Ziel helfen. Der einfache Zugang zu postgradualen Studiengängen im Bereich Rechnungswesen bietet dem Einzelnen die Möglichkeit, sich neben dem Markt zu positionieren, um einen Beitrag zu Unternehmen, Wirtschaft und Gesellschaft zu leisten.

\subsection{WIRTSCHAFTLICHE ENTWICKLUNG UND WACHSTUM}

Furtado (1964) definiert wirtschaftliche Entwicklung als "einen Prozess des sozialen Wandels, durch den eine zunehmende Anzahl menschlicher Bedürfnisse durch eine Differenzierung im Produktionssystem infolge der Einführung technologischer Innovationen befriedigt wird".

Für Hewlett (1981) wird wirtschaftliche Entwicklung definiert als "eine signifikante Erhöhung des realen Pro-Kopf-Einkommens einer Nation, mit dem Ziel, bessere Nahrung, Gesundheit, Bildung, bessere Lebensbedingungen und ein immer breiteres Spektrum an Arbeits- und Freizeitmöglichkeiten für die Menschen dieser Nation zu erhalten".

Auf der anderen Seite steht ein Wirtschaftswachstum, das nach Siedenberg (2006) aus einem "Prozess von Veränderungen überwiegend quantitativen Charakters, also einer Zunahme von Größe, Volumen und/oder Menge" besteht. Zusammenfassend, was Vasconcellos (2000) schlussfolgerte, wies darauf hin, dass Wirtschaftswachstum das kontinuierliche Wachstum des Pro-Kopf-Einkommens über eine Zeit ist.

Das Wachstum und die Erhaltung des Bruttoinlandsprodukts (BIP) fördern eine höhere Lebensqualität der Bevölkerung. Darüber hinaus sind technologische Innovationen, höhere Staatseinnahmen, die Erweiterung der Belegschaft und Einsparungen weitere Faktoren, die zum Wirtschaftswachstum eines Landes beitragen.

Es wird beobachtet, dass, wenn ein Land Wirtschaftswachstum zeigt, die Zahl der Arbeitslosen abnimmt, während die erhobenen Steuern steigen. In diesem 
Zusammenhang müssen mehr investiert werden, um das anhaltende Wachstum der Wirtschaft aufrechtzuerhalten. Mendes (2015) erklärt, dass sich die Wirtschaft und das Wachstum der Eltern aufgrund einiger Faktoren, einschließlich Bildung und hoher Steuerbelastung, unterdurchschnittlich entwickelt haben.

Im Einklang mit dem Wirtschaftswachstum kommt die wirtschaftliche Entwicklung, dass es für das Land notwendig ist, Indikatoren beizubehalten und Faktoren zur Verbesserung der Lebensqualität und des Einkommens der Bevölkerung im Laufe der Zeit zu haben. Für Rocha (2004) zeigt dies: das Wachstum des wirtschaftlichen Wohlergehens, gemessen an wirtschaftlichen Indikatoren, unter denen es gültig ist, das gesamte Nationalprodukt und das Nationalprodukt pro Kopf zu erwähnen; Verringerung der Armut, der Arbeitslosigkeit und des sozialen Lebens; und die Verbesserung der Gesundheits-, Ernährungs-, Bildungs- und Wohnbedingungen.

Der Human Development Index - HDI der Vereinten Nationen (UN) ist eine Maßeinheit, die erstellt und verwendet wird, um den Entwicklungsstand eines bestimmten Landes zu überprüfen, der Gesundheit, Bildung und Einkommen umfasst. Der Index ist eine numerische Referenz, deren Variation zwischen 0 und 1 definiert ist. Je näher der Wert an 1 liegt, desto besser sollte die Entwicklung dieses Ortes in den präsentierten Elementen sein. Kein Land hat einen HDI von null oder gar eins (FILGUEIRA et al., 2010).

Laut dem UN-Bericht, der die Bewertung von 189 Ländern enthält, erreichte Brasilien im Jahr 2018 0,761 Punkte, was einen deutlichen Anstieg im Vergleich zu 2013 darstellt, wo es 0,752 erhielt.

Brasiliens Position ist 79. in der Rangliste, neben Kolumbien und rangiert unter den Ländern mit hoher menschlicher Entwicklung Kategorie. Im Bericht ist der höchste Indikator der von Norwegen mit 0,954 Punkten, und mit dem schlechtesten Index für die menschliche Entwicklung ist Niger mit 0,377 (LEUSIN JÚNIOR, 2015).

\subsection{NEUE TECHNOLOGIEN IN DER BUCHHALTUNG}


Neue Technologien erzeugen mit ihrer Anwendung strukturelle Veränderungen in Organisationen, wirken sich auf die Kosten der Unternehmen aus und modifizieren ihre Produktionsprozesse im Hinblick auf die Wettbewerbsfähigkeit auf dem Markt (MAT, 2010).

Scott (2009) betont, dass der Einsatz von Technologie in der Buchhaltung eine beispiellose Veränderung ist, dh es ist nicht einfach zu erkennen, ob ihre Auswirkungen negativ oder positiv waren. Aus einem anderen Blickwinkel zeigen Alsharayri (2011) und Choe (2004), dass das Niveau der produzierten Technologie und Informationen positive Auswirkungen hat.

Technologie hat positive Auswirkungen sowohl auf die Agilität und Zuverlässigkeit als auch auf die Sicherheit von Informationssystemen, die in Unternehmen von Buchhaltungsbüros und Fachleuten verwendet werden. Die Funktion der von den Systemen generierten Informationen besteht laut Allahyari und Ramazani (2011) darin, ihren Nutzern zu helfen, Entscheidungen zu treffen, da die Buchhaltung in der Lage ist, Informationen zu produzieren, die für die Formulierung von Geschäfts- und strategischen Prozessen, für die Kontrolle von Aktivitäten und für das Wachstum von Unternehmen relevant sind.

Für Scott (2009) liegt der Vorteil der Technologie in der Verwendung mehrerer Werkzeuge, um die Ausführung der vom Zähler ausgeführten Funktionen zu beschleunigen. In diesem Aspekt trägt die Technologie angesichts der Geschwindigkeit und Effizienz, die der technologische Fortschritt bietet, zur operativen Weiterentwicklung der Rechnungslegung angesichts der Wettbewerbsfähigkeit bei.

Acevedo (2012) zeigt, dass, wenn die Kommunikation in Buchhaltungsunternehmen schnell ist, sie dazu beitragen können, die Produktivität zu steigern, die Entscheidungsfindung zu verbessern und das Geschäftswachstum zu erleichtern.

Auch nach Scott (2009) ist ein Nachteil der Technologie ihre Abhängigkeit vom Menschen. Technologie entsteht aus menschlicher Innovation, daher ist es ein Produkt, das die gleichen menschlichen Fehler wiederholt. Dies kann jedoch mit 
robusteren Prozessen und mit einer gezielteren Spezialisierung und Ausbildung zur Automatisierung umgangen werden. Es zeigt auch, dass ein weiterer Nachteil die Abhängigkeit des Berufs von der Technologie ist, aber es ist bereits bekannt, dass Studien zeigen, dass der Zähler, der existierte, um Berechnungen zu operationalisieren, aussterben wird, was diesem falschen Gedanken des Autors widerspricht.

Aribaba et al. (2011) wiederum heben die Bedeutung hervor, die Technologie für die Gesellschaft, insbesondere in kleinen Dienstleistungsunternehmen, angesichts der Verbesserung der Leistung und der größeren Geschäftsentwicklung hinzufügt. So entstehen mit mehr Leistung mehr Chancen und Gewinne, damit werden Arbeitsplätze geschaffen und die Wirtschaft profitiert.

Wettbewerbsfähigkeit erfordert, dass Buchhaltungsorganisationen in der Lage sind, Wert für ihre Kunden zu schaffen. Diese Kunden, die Steuerzahler und Auftragnehmer sind. Laut Simons (1987) werden diese Organisationen durch die Differenzierung von ihren Mitbewerbern, die Implementierung und Formulierung einer klar definierten Geschäftsstrategie mehr Ergebnisse erzielen. In der Strategie ist der Einsatz von Technologien, die den Buchhaltungssystemen hinzugefügt werden, von wesentlicher Bedeutung, da sie sichere und nützliche Informationen liefern (JERMIAS; GANI; 2004).

Eine klar definierte und konsolidierte Strategie, die auf Investitionen in Technologie und Qualifikation der Menschen basiert, wird laut Grande et al. (2011) produktive Vorteile und günstige Veränderungen für seine Kunden im Vergleich zu seinen Wettbewerbern bringen.

\subsection{ROLLE DES BUCHHALTERS BEI DER INNOVATION UND AUTOMATISIERUNG VON BUCHHALTUNGSPROZESSEN}

Die Zahl der Buchhaltungsbüros steigt und trägt zum Rückgang der Arbeitslosigkeit zwischen den Jahren 2009 und 2012 bei, so Gondim (2014). Es gab einen Anstieg von mehr als $60 \%$ der formellen Arbeitsplätze und $5 \%$ bei neuen Wirtschaftsprüfungsgesellschaften. 
Der Eidgenössische Rechnungslegungsrat (CFC, 2014) veröffentlicht auf seiner Website eine durchgeführte Umfrage, die zeigt, dass mehr als 58\% der Fachleute im Bereich Rechnungswesen lieber in privaten Unternehmen arbeiten oder ein eigenes Büro eröffnen, als die Prüfung ablegen zu müssen und in den öffentlichen Sektor einsteigen. Beachten Sie, dass diese Daten den Beitrag zur Schaffung einer Vielzahl formeller und informeller Arbeitsplätze zeigen, da der Buchhalter mit den Managern der Unternehmen (deren Kunden) zusammenarbeitet, damit sich das Geschäft entwickelt und wächst und mehr Mitarbeiter benötigt.

Indem er Unternehmern hilft oder in sein eigenes Unternehmen investiert, indem er praktisches und theoretisches Wissen verwendet, spielt der Buchhalter eine bedeutende Rolle in der Wirtschaft. In den letzten Jahren hat die Buchhaltung aufgehört, nur einen Taschenrechner und Unmengen von Papier zu verwenden (NOGUEIRA, 2010).

Sehen Sie, dass Coelho (2015) erklärt, dass der Buchhalter von der Person, die vergangene Fakten aufzeichnet, zu einem strategischen Berater mit einer prospektiven und breiten Sicht auf den Markt wurde. Basierend auf diesen Veränderungen hat der Schütze die Mission, Prozesse zu automatisieren, mehr Leistung zu generieren und statistische Daten zu komprimieren, um Unternehmen und Manager bei der Entscheidungsfindung zu unterstützen.

Fávero et al. (2006) zeigen, dass "die Buchhaltung darauf abzielt, Informationen für die verschiedenen Benutzer zu generieren, damit sie Entscheidungen treffen können". Dieses Ziel scheint einfach, aber die subjektiven Faktoren, die daran beteiligt sind, machen diesen Prozess schwierig, die Schwierigkeit, die Daten zu verstehen und die Distanzierung des Buchhalters des Managers machen es schwierig, dieses Ziel zu erfüllen. Lassen Sie uns sehen, dass es Variablen für eine gute Kommunikation gibt, und diese müssen studiert werden und die Spezialität des Buchhalters, damit sein Ziel als Buchhaltungsprofi erreicht wird.

Da eine Zielgruppe von Buchhaltungsinformationen die neue Generation identifiziert, die zuverlässige Informationen benötigt und verlangt, dass sie schnell sind, gegen 
Drucker (1987), besteht die spezifische Aufgabe des Unternehmermanagers eines Unternehmens darin, das Geschäft dieses Unternehmens agiler und qualitativ hochwertiger zu machen.

Laut daten, die auf der CFC-Website (2014) verfügbar sind, hat Brasilien derzeit mehr als 71.008 Buchhaltungsorganisationen / -unternehmen und 515.011 Fachleute, die beim Federal Accounting Council registriert sind, und diese Fachleute und Büros müssen auf die Automatisierung von Prozessen vorbereitet sein.

Da der Buchhalter die Funktion hat, Manager zum Wachstum ihrer Unternehmen zu führen, spielt dieser Fachmann auch eine wichtige Rolle in der Gesellschaft und sollte daher immer auf der Suche nach der Aktualisierung seines Wissens und seines ethischen Verhaltens sein, als Gesprächspartner der Regierung, mit dem Steuerzahler und der Gesellschaft (CORONADO, 2017).

Der Buchhalter, der seine Kunden anleitet, richtig beizutragen, hilft bei der Entwicklung des Staates und damit bei der Entwicklung des Landes. In dieser Rolle ist die Automatisierung der Schlüssel zur gesamten Aufmerksamkeit des Buchhalters in der Generierung von Informationen und nicht in langweiligen und zeitaufwändigen Aufgaben von Konferenzen und Releases.

Kompetenz, Professionalität, Agilität und Spezialisierung der an den Prozessen Beteiligten machen sie kohärenter, agiler und funktionieren richtig. Effiziente und spezialisierte Buchhaltungspraxis ist der Punkt, der auf die Geschäftserhaltung geachtet werden sollte und zunehmend effizient und wettbewerbsfähig ist.

Der Fachmann, der diese Verhaltensänderungen versteht, die passiert sind, sich spezialisiert und aktualisiert, wird eine wichtige und entscheidende Rolle in der Gesellschaft und in Unternehmen spielen. Der gesamte Prozess hängt von einem Menschen ab, und dieser Mensch muss die Fähigkeiten und Fähigkeiten haben, damit der automatisierte Prozess richtig funktioniert, zusätzlich zu einem systemischen Wissen über Unternehmen. Spezialisierung und fundiertes Wissen sind Werkzeuge für dieses neue Paradigma des Wandels im Buchhaltungsberuf. 


\section{FAZIT}

Wie in diesem Artikel erwähnt, ist der Beruf des Buchhalters von Anfang an wichtig für die Entwicklung und das Wachstum des Landes. Die Automatisierung der Buchhaltungsprozesse und die Änderung der Anforderungen des Berufsstandes werden den Fachmann, der heute nur als der Erfüllungsgesetz angesehen wird, erlöschen lassen. Dann bietet sich für den Beruf und die Fachleute die Möglichkeit, sich in anderen Bereichen ihres Wissens wie Beratung und Geschäftsstrategie abzuheben und das umfangreiche Wissen, das in der Ausbildung erworben wurde, für die Ausbildung einzusetzen.

Buchhaltungsbüros tragen zur Generierung von Arbeitsplätzen bei und erfüllen so ihre soziale Rolle, müssen sich jedoch an die neue Dynamik von Informationssystemen anpassen, damit sie effizient sind und mit Qualität und Agilität die für Unternehmen und Regierungen notwendigen Informationen produzieren. Da Bildung eine Grundlage für die Entwicklung jeder Nation ist, bringt die Verbesserung des Berufs und der Ausbildung des Fachmannes eine signifikante Verbesserung der Basis der Gesellschaft und damit Ereignisse der sozialen und beruflichen Verbesserung mit sich.

Der Buchhaltungsprofi ist nicht mehr derjenige, der nur schlechte Nachrichten an Unternehmen bringt, mit Leitfäden und Kreditorenbuchhaltung, Unternehmern und Managern eine strategische Orientierung bietet und dazu beiträgt, dass sie ihre Funktionen korrekt und mit der Agilität ausführen, die der Markt durch korrekte Daten erfordert und schnell extrahiert.

Contador verfügt über alle Werkzeuge und Kenntnisse, um die Erstellung von Abschlüssen und Veröffentlichungen zu automatisieren, um mehr Agilität und Zuverlässigkeit zu bringen, und entwickelt sich somit zu einem Verbündeten für Unternehmen, um ihre soziale Rolle beim Geschäftswachstum zu spielen.

Unternehmen, die solide und intelligent wachsen und ihre Steuer- und Unternehmenspflichten mit Qualität und Agilität erfüllen, eine Rolle, die der Buchhalter zusammen mit dem Management spielen kann, werden die gesamte Gesellschaft 
entwickeln lassen. Wenn jeder seine Rolle in Wirtschaft und Gesellschaft erfüllt, werden neue Arbeitsplätze geschaffen, mehr Steuern gezahlt, die Armutsraten sinken und das Bildungsangebot wird höher sein.

Die Gefahr der Nichtexistenz des Berufs wird dazu führen, dass sich Fachleute neu erfinden und ihre Position angesichts von Innovationen überprüfen. Diejenigen, die eine andere Sicht auf ihre Position als Buchhaltungsprofi haben, kombiniert mit neuen Technologien der künstlichen Intelligenz, Automatisierung und Spezialisierung, haben Platz auf dem Markt gewonnen, anders als der Profi, der die Veränderungen nicht wahrnäht und gegen sie kämpft.

Auf der anderen Seite muss die Buchhaltungsausbildung verbessert werden, damit neue Technologien in der Lehre eingesetzt werden und die Auszubildenden die Universitäten verlassen, um sich der aktuellen Welt zu stellen, die Agilität und Qualität bei der Erbringung von Dienstleistungen erfordert.

Angesichts des Innovationsgrades von Unternehmen in bestimmten Regionen ist eine $\mathrm{Zu}$ beobachtende Lücke in Bezug auf die demografische Verteilung der Buchhaltungsfachleute. Dies gibt einen breiteren Überblick darüber, was mit der Karriere passieren kann oder nicht. Es wird auch empfohlen, Fallstudien in innovativen Unternehmen mit einem hohen Automatisierungsgrad und der Positionierung des Fachmanns gegenüber diesem Unternehmen und seinen Ergebnissen durchzuführen. Diese Studien werden zu einem breiteren Verständnis dessen beitragen, was in Zukunft passieren kann.

\section{VERWEISE}

ABREU, E. N. de. Regulamentação de auditoria: estudo da influência das fraudes contábeis de 2001. Goiânia: Universidade Federal de Goiás - UFG, 2013.

ACEVEDO, L. Business benefits of information technology. Chron, 2019. Disponível em: <http://smallbusiness.chron.com/business-benefits-informationtechnology-4021.html >. Acesso em: 10/mai/2021 
ALLAHYARI, A.; RAMAZANI, M. Firm technological change and its effects on management accounting change: case study of Iranian manufacturing firms. Global Journal of Management and Business Research, v. 11, n. 9, 2011.

ALSHARAYRI, M. A. The E-Commerce impact on improving accounting information system in Jordanian Hotels. International Research Journal of Finance and Economics, v. 75, 2011.

ARAÚJO, F. M. de. A inteligência artificial e os seus impactos no mundo do trabalho. Fortaleza: Centro Universitário Fametro, 2020.

ARIBABA, F. et al. An evaluation of the impact of technological innovative entrepreneurial development programmes on the performance of small scale business in Nigeria. Global Journal of Business, Management And Accounting, v. 1, n. 1, 2011.

CARVALHOSA, M. de S. B. Comentários à lei de sociedades anônimas. Saraiva Educação SA, Editora: Saraiva Educação S. A., v. 1. 2017.

CFC. Conselho Federal de Contabilidade. Dados estatísticos do Conselho Federal de Contabilidade. 2014. Disponível em: http://www.cfc.org.br/. Acesso em: 12/mai/2021.

CHIAVENATO, I. Empreendedorismo: dando asas ao espírito empreendedor. Editora manole, $4^{\mathrm{a}}$ ed. 2004.

$\mathrm{CHOE}, \mathrm{J}$. Impact of management accounting information and $A M T$ on organizational performance. Journal of Information Technology, v. 19, 2004.

COELHO, J. M. A. Contabilidade: uma carreira em transformação. Disponível em: http://www.portalcfc.org.br/noticia.php?new=23196. Acesso em: 12/mai/2021.

CORDEIRO, C. M. R. Auditoria e Governança corporativa. Editora: IESDE, 2011. 
CORONADO, O. Contabilidade gerencial básica. Editora: Saraiva Educação SA, 2017.

CUNHA, E. S. Oferta de crédito e emprego local nos municípios da região Nordeste: 2002-2015. Salvador: Federal da Bahia. 2019.

D'AURIA, F. Primeiros princípios de contabilidade pura. São Paulo: Departamento de Cultura e Ação Social, 1949.

DE OLIVEIRA REIS, A. et al. Perfil do profissional contábil: habilidades, competências e imagem simbólica. Revista Contemporânea de contabilidade, v. 12, n. 25, p. 95-116, 2015.

DINIZ, E. R. da S. et al. Governança eletrônica no Brasil e o papel das tecnologias da informação e comunicação. Florianópolis: Universidade Federal de Santa Catarina, 2014.

DOS SANTOS, M. J. A. et al. Um estudo comparativo entre o exame de suficiência do conselho federal de contabilidade, o exame nacional de desempenho de estudantes e a pós-graduação stricto sensu dos cursos de ciências contábeis do Estado do Rio de Janeiro. Polêm!ca, v. 13, n. 4, p. 1681-1698, 2014.

DRUCKER, P. F. Inovação e Espírito Empreendedor: prática e princípios. São Paulo: Pioneira, 1987.

FAVERO, H. L. et al. Teoria e Prática. 4 ed. Editora: Atlas, São Paulo. 2006.

FIGUEIREDO, C. R.; STRASSBURG, U. A Contabilidade: aspectos históricos que influenciaram no seu desenvolvimento no decorrer do tempo. IX Jornada de Estudos Contábeis-UNIOESTE, 2008.

FILGUEIRA, J. M. et al. Um panorama estatístico de cunho econômico, social e educacional do estado do Rio Grande do Norte. HOLOS, v. 4, p. 198-215, 2010. 
FURTADO, C. Dialética do Desenvolvimento. Rio de Janeiro, $2^{a}$ ed., Fundo de Cultura, 1964.

GIL, A. C. Métodos e técnicas de pesquisa social. 6. ed. São Paulo: Atlas, 2008.

GONDIM, A. MEI também tem obrigações fiscais. Contábeis, 2016. Disponível em: https://www.contabeis.com.br/noticias/26714/mei-tambem-tem-obrigacoes-fiscais/.

GRANDE, E. U. et al. The impact of accounting information systems (AIS) on performance measures: empirical evidence in Spanish SMEs. The International Journal of Digital Accounting Research, v. 11, 2011.

HEWLETT, S. A. Dilemas do desenvolvimento. Rio de Janeiro: Zahar, 1981.

IUDÍCIBUS, S. de; MARION, J. C. Introdução à teoria da contabilidade para o nível de graduação. 4 ed. São Paulo. Editora: Atlas, 2006.

JERMIAS, J.; GANI, L. Integrating business strategy, organizational configurations and management accounting systems with business unit effectiveness: a fitness landscape approach. Management Accounting Research, v. 15, 2004.

LEITE, E. F. O fenômeno do empreendedorismo. Saraiva Educação SA, 2017.

LEUSIN JÚNIOR, S. A inserção internacional de potências médias: evidências da afirmação de países em desenvolvimento a luz da evolução do sistema multilateral de comércio. Porto Alegre: Universidade Federal do Rio Grande do Sul, 2015.

MARCONI, M. de A.; LAKATOS, E. M. Metodologia do trabalho científico. São Paulo: Editora Atlas, 1992. 4a ed.

MAT, T. Z. Management accounting and organizational change: impact of alignment of management accounting system, structure and strategy on 
performance. Philosophy School of Accounting, Finance and Economics Faculty of Business and Law Edith Cowan University. Perth Western Austrália, 2010.

MENDES, M. Por que a economia do Brasil foi para o buraco? Brasil economia e governo, 2015. Disponível em: http://www.brasil-economiagoverno.org.br/2015/08/25/por-que-a-economia-brasileira-foi-para-o-buraco/. Acesso em: 14/mai/2021.

NETO, O. R. M. A profissionalização do contador no Brasil. São Paulo: Mackpesquisa, $2010 . \quad$ Disponível em: http://168.197.92.160/bitstream/handle/10899/14605/721_2008_0_15.pdf?sequence $=1$ \&isAllowed=y/. Acesso em: 14/mai/2021.

NOGUEIRA, M. Práticas de contabilidade: relatório de estágio do Mestrado em Gestão. Coimbra: [s.n], 2010.

RAMALHO, T. C. L. Contabilidade criativa: a percepção dos Técnicos Oficiais de Contas. Lisboa: Instituto Superior de Contabilidade e Administração de Lisboa. 2015.

ROCHA, F. J. M. Educação e economia: uma abordagem sobre as consequências e condicionantes econômicas do desenvolvimento humano, com ênfase em educação. Brasília: Cadastro de Finanças Públicas, n. 5, 2004.

ROZENBAUM, S. Revolução digital deixa cinco profissões à beira da extinção. rfi, 2018. Disponível em: https://www.rfi.fr/br/economia/20180829-revolucao-digitaldeixa-cinco-profissoes-beira-da-extincao-diz-estudo.

SANTOS, R. F. dos. Introdução à contabilidade: noções fundamentais. São Paulo: Saraiva, 2008.

SCOTT, W. The impact technology is having on the accounting profession. World conference on higher education, 2009. 
SIEDENBERG, D. R. Dicionário do Desenvolvimento Regional. Santa Cruz: Edunisc, 2006.

SIMONS, R. Accounting control systems and business strategy: an empirical analysis. Accounting, Organizations and Society, v. 12,1987.

SOUZA, E. C. et al. As características qualitativas da informação contábil a partir da percepção dos profissionais de contabilidade. In: Congresso UFPE de Ciências Contábeis. 2018.

VASCONCELLOS, M. A. S. de. Economia Micro e Macro: Teoria e Exercícios, Glossário com 260 Principais Conceitos Econômicos. São Paulo: Atlas, 2000.

Eingereicht: August 2020.

Genehmigt: Juni 2021. 